\title{
A Case of Life-threatening Actinomyces turicensis Bacteremia
}

Tikal Kansara $^{1}$, Monil Majmundar ${ }^{1}$, Rajkumar Doshi ${ }^{2}$, Kuldeep Ghosh ${ }^{1}$, Mohammad Saeed ${ }^{1}$

1. Internal Medicine, New York Medical College-Metropolitan Hospital Center, New York City, USA 2. Internal Medicine, University of Nevada, Reno School of Medicine, Reno, USA

Corresponding author: Tikal Kansara, tikal_kansara@yahoo.co.in

\begin{abstract}
Actinomyces turicensis (A. turicensis) are normal commensals of the oral, gut, vagina, and skin flora. Infection with these organisms is usually benign, and bacteremia is rare. Here, we describe a case of an otherwise healthy female patient presenting with renal calculi and life-threatening $A$. turicensis bacteremia. The patient did not have any risk factors for $A$. turicensis bacteremia. The patient developed multi-organ dysfunction syndrome and received a biodegradable right ureter stent. The patient improved with urosurgical intervention and appropriate antibiotic coverage.
\end{abstract}

Categories: Internal Medicine, Urology, Infectious Disease

Keywords: actinomyces turicensis, bacteremia

\section{Introduction}

Actinomyces are gram-positive, facultative anaerobic bacilli and are normal commensals of the oral, gut, vagina, and skin flora [1]. In the majority of cases, the infection with these organisms goes unnoticed [2]. This is mostly due to the natural immunity of the body as well as the organism being susceptible to commonly available antibiotics [1]. Bacteremia with these organisms is rare. Localized Actinomyces infection can mimic malignancy, nocardiasis or cold abscess [2]. The majority of Actinomyces bacteremia patients described in the literature had an abscess or severe infection, to begin with, or were immunocompromised [3]. There are no reported cases of Actinomyces turicensis (A. turicensis) bacteremia in patients without any predisposing risk factors. We discuss a case of $A$. turicensis bacteremia in an otherwise healthy patient.

Received 01/14/2020

Review began 01/20/2020 Review ended 01/21/2020 Published 01/24/2020

\section{() Copyright 2020}

Kansara et al. This is an open access article distributed under the terms of the Creative Commons Attribution License CC-BY 3.0., which permits unrestricted use, distribution, and reproduction in any medium, provided the original author and source are credited.

\section{Case Presentation}

A 52-year-old woman presented with concerns of lower abdominal and flank pain for three days. She had associated loss of appetite, vomiting, nausea, and low-grade intermittent fever. She denied hematuria, dysuria, diarrhea, or trauma. She had no chronic medical illness. Four years ago, she had an emergency department (ED) visit for urinary tract infection and two years prior for vaginitis. She was allergic to penicillin which causes a rash. On physical examination, her blood pressure was $92 / 55 \mathrm{mmHg}$, she was drowsy, responding to deep pain stimulus, and occasionally responding to verbal commands with mild right lower abdominal tenderness.

Urinalysis showed 10 to 15 white blood cells (WBCs) per high-power field, moderate leucocyte esterase, and moderate blood in the urine. Complete blood counts revealed leukocytosis of $12.22 \mathrm{k} \mathrm{WBC} / \mathrm{mcL}$ (normal: 4.5k-11.0k WBC/mcL) with neutrophilia $88.1 \%$, minimally elevated creatinine at $1.2 \mathrm{mg} / \mathrm{dL}$ (normal: 0.4-1.6 $\mathrm{mg} / \mathrm{dL}$ ) with a glomerular filtration rate of $47 \mathrm{~mL} / \mathrm{min} / 1.73 \mathrm{~m}^{2}$ (normal: $>60 \mathrm{~mL} / \mathrm{min} / \mathrm{m}^{2}$ ), and elevated transaminases with aspartate transaminase of $66 \mathrm{U} / \mathrm{L}$ (normal: 10-35 U/L) and alanine transaminase of 55 $\mathrm{U} / \mathrm{L}$ (normal: 7-45 U/L). A computed tomography scan of the abdomen and pelvis showed right-sided hydronephrosis (Figure 1) and proximal hydroureter secondary to a linear stone measuring $6 \mathrm{~mm}$ in the proximal right ureter (Figure 2). Also, right-sided perinephric stranding and bilateral non-obstructing kidney stones-two on the right (Figure 3) and one on the left measuring $4 \mathrm{~mm}$ (Figure 1)-were noted. The uterus was enlarged with multiple calcified and non-calcified masses representing myoma. The results of other investigations including coagulation panel, hepatic panel, amylase, and lipase, were normal. Urine culture and two sets of blood cultures were drawn. She was given ceftriaxone empirically in the ED. 


\section{Cureus}

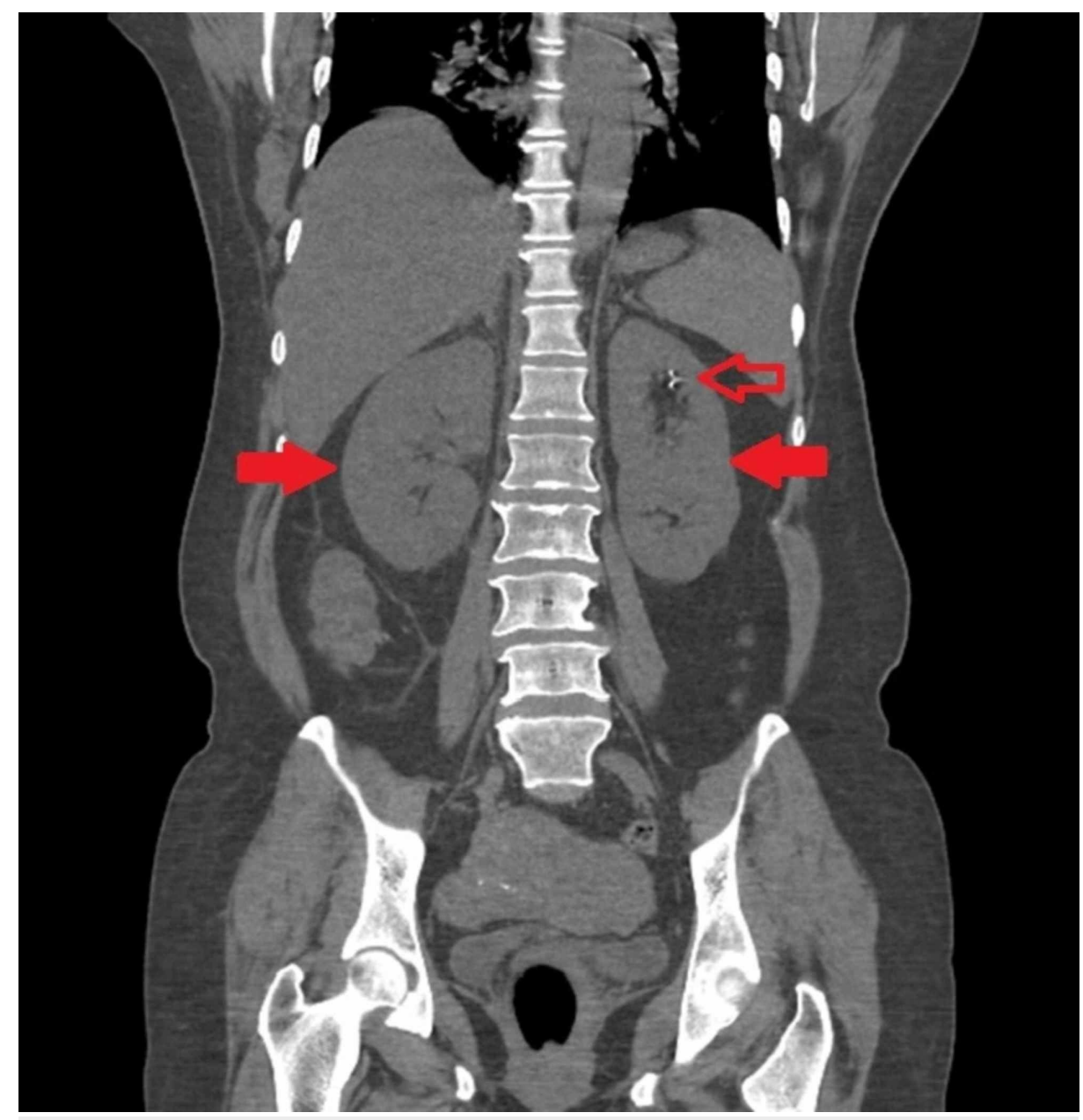

FIGURE 1: Computed tomography of the abdomen with contrast showing bilateral hydronephrosis (solid red arrows) with left nonobstructing renal calculi (red arrow) 


\section{Cureus}

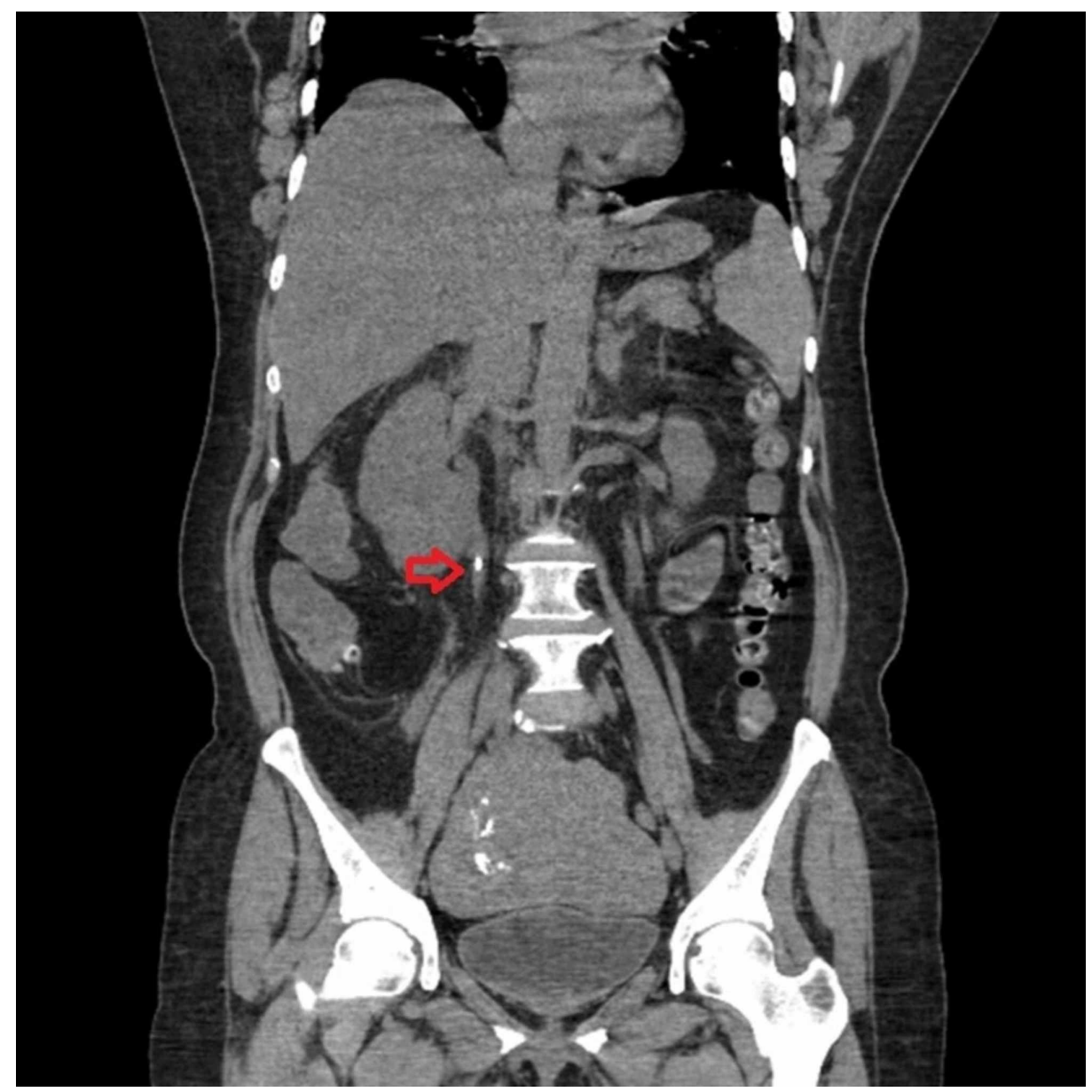

FIGURE 2: Computed tomography of the abdomen with contrast showing right-sided proximal ureteric renal calculi (red arrow) 


\section{Cureus}

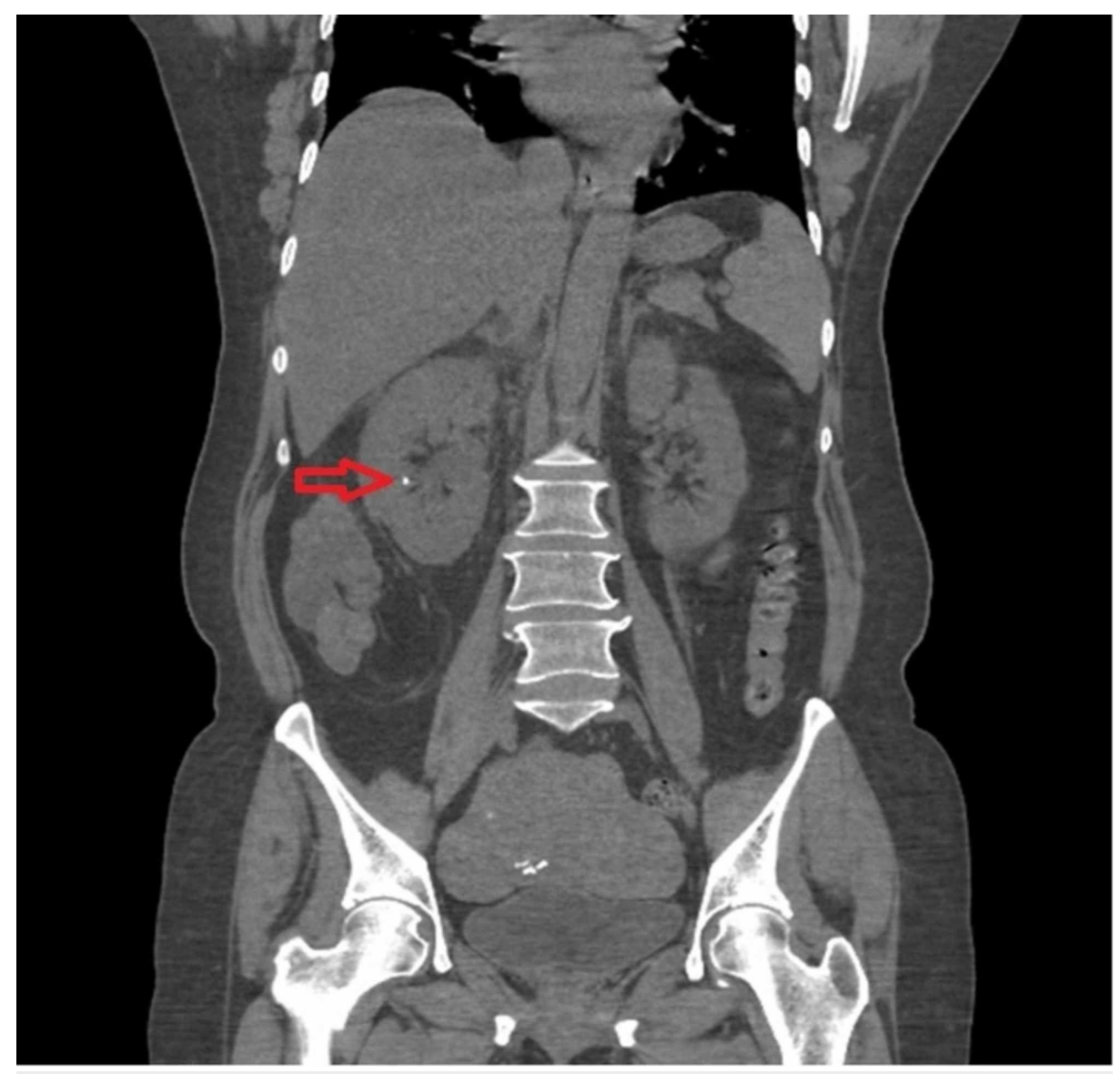

\section{FIGURE 3: Computed tomography of the abdomen with contrast showing non-obstructive right renal calculi (red arrow)}

A diagnosis of septic shock with multi-organ dysfunction syndrome in the setting of urinary tract infection with impending pyelonephritis and abscess was made. The patient had to be admitted to the medical intensive care unit (MICU). She was started on intravenous vancomycin and meropenem and scheduled for ureteric stent placement. A right ureteric biodegradable stent was placed. A foley catheter was placed postoperatively to aid in recovery of left-sided hydronephrosis. Within the next few hours, the patient regained consciousness and was deemed stable. She was transferred out of the MICU, where she had hematuria and lower abdominal pain for two days, likely due to stent placement, which resolved completely. She was continued on vancomycin and meropenem for another nine days.

The blood cultures drawn on admission were found be positive for $A$. turicensis sensitive to penicillin, tetracycline, clindamycin, and ampicillin on the fourth day. Urine culture was negative for any growth. Based on culture and sensitivity, ceftriaxone was started, as the patient was allergic to penicillin, and continued for six days, for a 15-day regimen of antibiotics. The patient was discharged to home on 10th day with a regular follow-up in the clinic for general health care maintenance. She had no further symptoms. Her stent was removed in an outpatient setting. Culture of the removed stent did not grow A. turicensis organism.

\section{Discussion}

The Actinomyces species are facultative anaerobic gram-positive bacilli with a filamentous appearance [1]. Actinomyces are common inhabitants of the gut and genitourinary tract [3]. In women, Actinomyces commensals include A. meyeri, A. neuii, A. radingae, A. turicensis, and A. urogenitalis [1,3-5]. The organism resides on mucosal surfaces and gains access to deeper tissues via trauma, surgical procedures, or foreign bodies, which disrupt the mucosal barrier [3]. Human actinomycosis is a chronic granulomatous infectious disease caused by Actinomyces species [3]. Typical actinomycosis is characterized, according to the body site, as orocervicofacial, thoracic, and abdominopelvic forms $[6,7]$. Abdominopelvic actinomycosis is usually a consequence of invasive procedures such as cholecystectomy and intrauterine contraceptive devices [7-11]. Abdominopelvic actinomycosis in women with A. turicensis is common in connection with urethritis, cystitis, adnexitis, endometritis, cervicitis, vaginitis, and vulvitis [12]. Our patient had urinary tract infection with septic shock, with two sets of blood cultures positive for Actinomyces, and a negative urine culture. 
overlooked [3]. There have been cases of actinomycosis abscess being misdiagnosed as tumors, only to later diagnose actinomycosis on histology of excised specimens [13]. On a thorough review of the literature, only a handful of cases of Actinomyces bacteremia were found. To our knowledge, this case is amongst the first 10 cases of $A$. turicensis bacteremia. Table 1 presents a summary of reported cases of $A$. turicensis bacteremia.

Summary of reported cases of $A$. turicensis bacteremia is as follows.

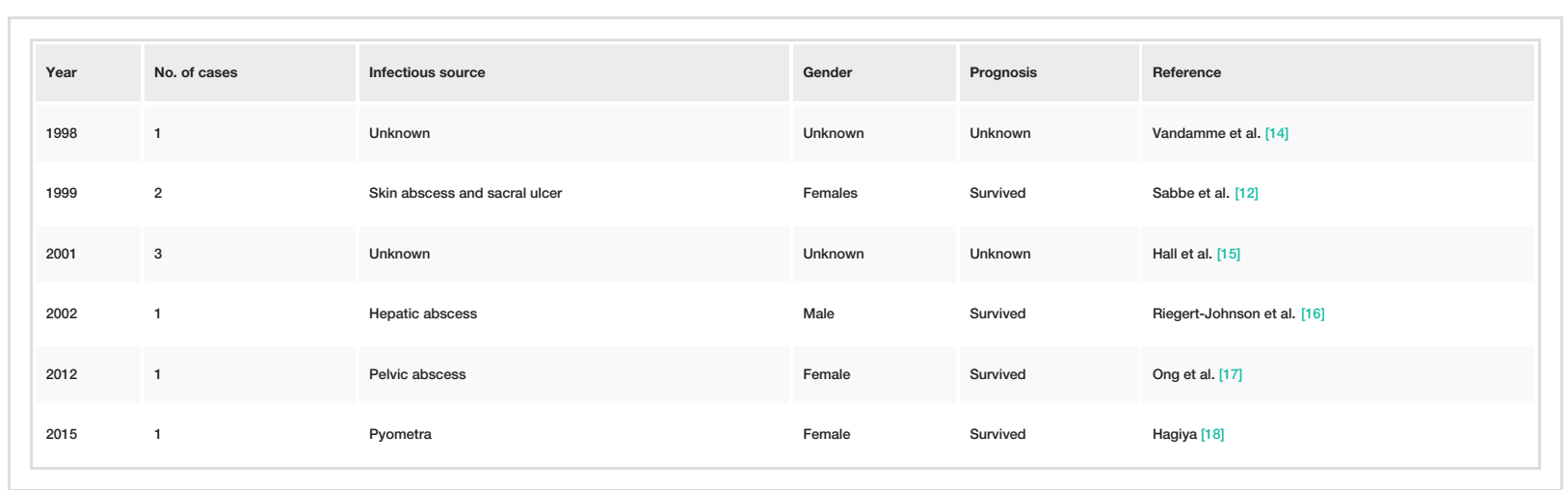

TABLE 1: All the reported cases of Actinomyces turicensis bacteremia

Of the nine reported cases of Actinomyces bacteremia, four were in female patients, one in a male patient, and gender was not described in the remaining. The clinical signs are subtle, and this organism can be diagnosed only by culture or advanced techniques. In most cases, Actinomyces is difficult to detect as it grows mostly as mixed flora and is susceptible to common empiric antibiotics. Furthermore, it grows slowly on blood agar, takes seven days to grow at $35^{\circ} \mathrm{C}[12]$. Histology of biopsied specimens, infected tissue, or pus is more sensitive than culture and can identify granulomatous inflammation, abscess, and necrosis along with sulfur granules in up to $75 \%$ of cases [18]. Immunofluorescence is highly specific for diagnosis. Molecular studies, such as 16S RNA sequencing, are a reference method for identification and classification. Polymerase chain reaction, with specific primers, could be used to identify microbial agents in clinical material $[12,18]$. None of these tests were performed for our patient because the organism was fully identified by blood cultures.

Actinomyces are generally susceptible to beta-lactam antibiotics and penicillin. Surgery and invasive procedures are considered in cases of extensive necrosis or failure of antibiotic therapy. Our patient was treated with vancomycin and meropenem initially until reports of culture were available, and then she was switched to ceftriaxone to complete 15 days of antibiotics. The patient improved significantly after the second day in the hospital, without any evidence of fever, hematuria, or abdominal pain which improved significantly after the second day of the hospital course.

\section{Conclusions}

A. turicensis bacteremia is usually a benign infection with a good prognosis as the organism is mostly sensitive to routine antibiotics, including beta-lactams. However, in some cases such as our patient, the infection can lead to septic shock and rapid clinical deterioration if untreated. Even though literature describes infection with Actinomyces species in immunocompromised patients, it can sometimes occur in otherwise healthy individuals as in our patient. Larger studies are required to determine association of $A$. turicensis infection with renal calculi. Fortunately, once the appropriate antibiotic is given, complete recovery is possible in a relatively short term.

\section{Additional Information}

\section{Disclosures}

Human subjects: Consent was obtained by all participants in this study. IRB BRANY issued approval N/A. Conflicts of interest: In compliance with the ICMJE uniform disclosure form, all authors declare the following: Payment/services info: All authors have declared that no financial support was received from any organization for the submitted work. Financial relationships: All authors have declared that they have no financial relationships at present or within the previous three years with any organizations that might have an interest in the submitted work. Other relationships: All authors have declared that there are no other relationships or activities that could appear to have influenced the submitted work.

\section{Acknowledgements}

The authors would like to acknowledge the contribution of Dr. Monica Pernia and Dr. Yoojin Kim for being a 
part of the management team of the case. The authors will also like to acknowledge the contribution of Dr. Giovanna Veruzza for helping in review of literature.

\section{References}

1. Hall V: Actinomyces-gathering evidence of human colonization and infection. Anaerobe. 2008, 14:1-7. https://doi.org/10.1016/j.anaerobe.2007.12.001

2. Valour F, Sénéchal A, Dupieux C, et al.: Actinomycosis: etiology, clinical features, diagnosis, treatment, and management. Infect Drug Resist. 2014, 7:183-197. https://doi.org/10.2147/IDR.S39601

3. Könönen E, Wade WG: Actinomyces and related organisms in human infections. Clin Microbiol Rev. 2015, 28:419-442. 10.1128/cmr.00100-14

4. Aila NAE, Tency I, Claeys G, et al.: Identification and genotyping of bacteria from paired vaginal and rectal samples from pregnant women indicates similarity between vaginal and rectal microflora. BMC Infect Dis. 2009, 9:167. 10.1186/1471-2334-9-167

5. Nikolaitchouk N, Hoyles L, Falsen E, Grainger JM, Collins MD: Characterization of Actinomyces isolates from samples from the human urogenital tract: description of Actinomyces urogenitalis sp. nov. Int J Syst Evol Microbiol. 2000, 50:1649-1654. 10.1099/00207713-50-4-1649

6. Santiago GLDS, Cools P, Verstraelen H, et al.: Longitudinal study of the dynamics of vaginal microflora during two consecutive menstrual cycles. PLoS ONE. 2011, 6:e28180. https://doi.org/10.1371/journal.pone.0028180

7. Smego JRA, Foglia G: Actinomycosis. Clin Infect Dis. 1998, 26:1255-1261. 10.1086/516337

8. Wong VK, Turmezei TD, Weston VC: Actinomycosis. BMJ. 2011, 343:6099-6099. 10.1136/bmj.d6099

9. Vyas JM, Kasmar A, Chang HR, Holden J, Hohmann E: Abdominal abscesses due to actinomycosis after laparoscopic cholecystectomy: case reports and review. Clin Infect Dis. 2007, 44:1-4. https://doi.org/10.1086/510077

10. Zbar AP, Ranasinghe W, Kennedy PJ: Subphrenic abscess secondary to Actinomycosis meyeri and Klebsiella ozaenae following laparoscopic cholecystectomy. South Med J. 2009, 102:725-727. 10.1097/SMJ.0b013e3181abddc5

11. Fiorino AS: Intrauterine contraceptive device-associated actinomycotic abscess and Actinomyces detection on cervical smear. Obstet Gynecol. 1996, 87:142-149. 10.1016/0029-7844(95)00350-9

12. Sabbe LJ, Van De Merwe D, Schouls L, Bergmans A, Vaneechoutte M, Vandamme P: Clinical spectrum of infections due to the newly described Actinomyces species A turicensis, A. radingae, and A. europaeus. J Clin Microbiol. 1999, 37:8-13.

13. Niknejad N, Moradi B, Niknezhad N, Safaei M, Nili F: Renal actinomycosis, a rare diagnosis which can clinically mimic a malignancy: case report and review of the literature. Arch Pediatr Infect Dis. 2018, 6:e13049. 10.5812/pedinfect.13049

14. Vandamme P, Falsen E, Vancanneyt M, et al.: Characterization of Actinomyces turicensis and Actinomyces radingae strains from human clinical samples. Int J Syst Bacteriol. 1998, 48:503-510. 10.1099/00207713-48$2-503$

15. Hall V, Talbot PR, Stubbs SL, et al.: Identification of clinical isolates of Actinomyces species by amplified 16S ribosomal DNA restriction analysis. J Clin Microbiol. 2001, 39:3555-3562. 10.1128/jcm.39.10.35553562.2001

16. Riegert-Johnson DL, Sandhu N, Rajkumar SV, Patel R: Thrombotic thrombocytopenic purpura associated with a hepatic abscess due to Actinomyces turicensis. Clin Infect Dis. 2002, 35:636-637. 10.1086/342327

17. Ong C, Barnes S, Senanayake S: Actinomyces turicensis infection mimicking ovarian tumour. Singapore Med J. 2012, 53:e9-e11.

18. Hagiya H: Pyometra perforation caused by Actinomyces without intrauterine device involvement . Case Rep Obstet Gynecol. 2013, 2013:1-2. 10.1155/2013/658902 